\title{
WORKSHOP
}

\section{THE EFFECT OF THE RETENTION LIMIT ON THE RISK RESERVE}

\author{
Maria de Lourdes Centeno* \\ Technical University of Lisbon
}

\begin{abstract}
This paper is devoted to the study of the initial reserve, as a function of the retention limit, needed to assure that the probability of ruin, at the end of a certain period of time, is not higher than an agreed value, for an excess of loss treaty. To assess the probability of ruin, the normal and the normal power approximation are used. It is shown that the initial reserve is not in general an increasing function of the retention, having a minimum under fair assumptions.
\end{abstract}

\section{KEYWORDS}

Risk reserve; excess of loss; normal power approximation; normal approximation.

\section{INTRODUCTION}

Consider a risk for which an excess of loss reinsurance treaty is sought. Let $M$ be the retention limit; $S=\sum_{i=0}^{N} X_{i}$, with $X_{0} \equiv 0$, the insurer's aggregate gross (of reinsurance) claims, in some fixed time interval, where $N$ is the number of claims and $\left\{X_{i}\right\}_{i=1, \ldots, N}$ are the individual claims; $P$ is the insurer's gross (of reinsurance) premium income with respect to the same risk.

Assume that the following assumptions are fulfilled:

$H_{1}: N$ is a mixed Poisson random variable, with

$$
\operatorname{Pr}\{N=k\}=\int_{0}^{\infty} \frac{\mathrm{e}^{-\lambda q}(\lambda q)^{k}}{k !} d H(q)
$$

where $\lambda$ is the expected value of $N$ and $H(q)$ denotes the distribution function of a random variable $Q$ with expected value equal to one, standard deviation $\sigma_{Q}$ and skewness coefficient $\gamma_{Q}$, where $\sigma_{Q} \geq 0$ and $\gamma_{Q} \geq 0$.

* Research performed under contract no. SPES-CT91-0063.

ASTIN BULLETIN, Vol. 25, No. 1, 1995 
$H_{2}:\left\{X_{i}\right\}_{i=1, \ldots, N}$ are i.i.d. non-negative random variables, independent of $N$, with common distribution function $G($.$) , absolutely continuous and such that$

$$
\left\{\begin{array}{cc}
G(x)=0 & x \leq 0 \\
0<G(x)<1 & x>0
\end{array}\right.
$$

$H_{3}$ : The expected value of $X_{i}$ exists and is denoted by $\mathrm{E}[X]$.

$H_{4}$ : The excess of loss reinsurance premium is calculated according to the expected value principle with loading coefficient $\alpha$, i.e. the loading is

$$
C(M)=\alpha \lambda \int_{M}^{\infty}(x-M) d G(x)
$$

with $\alpha>0$.

For an excess of loss reinsurance arrangement Beard, Pentikäinen and Pesonen (1984), p. 146, state the retention problem as the ascertainment of the maximum value of $M$ in such a way that it is granted, with probability $1-\varepsilon$, that the retained risk will not consume the initial reserve $U$ during the period under consideration. For the effect they have used the Normal Power approximation. An alternative reference to this problem is chapter 6 of Daykin, Pentikäinen and Pesonen (1994).

In the example given by them, with the data used through their book, the initial reserve is an increasing function with the retention limit.

It will be proved that this function is not always increasing, that under some fair assumptions it will have a minimum, which rends feasible the formulation of the problem as the determination of the retention limit in such a way that the initial reserve necessary to assure with probability $1-\varepsilon$ that is not absorbed during the period under consideration is reduced to a minimum.

\section{THE INITIAL RESERVE AS A FUNCTION OF THE RETENTION LIMIT}

Using the Normal Power approximation to the retained risk, with $z>1$, one gets (see, for instance, Beard, Pentikäinen and Pesonen (1984), p. 129)

$$
\begin{aligned}
U(M)= & z \sigma_{S}(M)+\frac{1}{6}\left(z^{2}-1\right) \sigma_{S}(M) \gamma_{S}(M)- \\
& -(P-\mathrm{E}[S]-C(M)), \quad M>0
\end{aligned}
$$

with $\Phi(z)=1-\varepsilon$, where $\Phi$ is the distribution function of a standard normal variable, and $\sigma_{S}(M)$ and $\gamma_{S}(M)$ denote respectively the standard deviation and the skewness coefficient of the retained risk $S(M)$,

$$
S(M)=\sum_{i=0}^{N} \min \left(X_{i}, M\right) .
$$


Let $a_{k}(M)$ be the $k$-th moment with respect to the origin of the retained claim amount, i.e.

$$
a_{k}(M)=\int_{0}^{M} x^{k} d G(x)+M^{k}(1-G(M))
$$

Then, having in mind that $S(M)$ follows a mixed compound Poisson distribution, the variance and the third central moment of $S(M)$ can be easily calculated (conditioning on $N$ ). They are

$$
\sigma_{S}^{2}(M)=\lambda a_{2}(M)+\lambda^{2} a_{1}^{2}(M) \sigma_{Q}^{2}
$$

and

$$
\mu_{3, S}(M)=\lambda a_{3}(M)+3 \lambda^{2} \sigma_{Q}^{2} a_{1}(M) a_{2}(M)+\lambda^{3} a_{1}^{3}(M) \gamma_{Q} \sigma_{Q}^{3}
$$

respectively, and hence the skewness coefficient $\gamma_{S}(M)$ is,

$$
\gamma_{S}(M)=\frac{\mu_{3, S}(M)}{\sigma_{S}^{3}(M)}
$$

It is possible to prove the result that follows.

\section{Result 1: If}

- the loading coefficient $\alpha$ satisfies

$$
\alpha>z \frac{\sigma_{N}}{\lambda}+\frac{1}{6}\left(z^{2}-1\right) \gamma_{N} \frac{\sigma_{N}}{\lambda},
$$

where $\sigma_{N}$ and $\gamma_{N}$ denote the standard deviation and the skewness coefficient of $N$,

- and the mixing distribution $Q$ has a skewness coefficient which is at most three times its variation coefficient, i.e. (having in mind that the mean of $Q$ is 1 )

$$
3 \sigma_{Q} \geq \gamma_{Q}
$$

then $U(M)$ given by (2) has a minimum.

Proof : Differentiating $\sigma_{S}(M), \sigma_{S}(M) \gamma_{S}(M)$ and $C(M)$ one gets

$$
\begin{gathered}
\sigma_{S}^{\prime}(M)=\sigma_{S}^{-1}(M)(1-G(M)) H(M), \\
\left(\sigma_{S}(M) \gamma_{S}(M)\right)^{\prime}=(1-G(M)) \sigma_{S}^{-4}(M) P(M)
\end{gathered}
$$

and

$$
C^{\prime}(M)=-\alpha \lambda(1-G(M))
$$

where

$$
H(M)=\lambda M+\lambda^{2} a_{1}(M) \sigma_{Q}^{2},
$$


and

(12)

$$
\begin{aligned}
P(M)= & \lambda^{2}\left(3 M^{2} a_{2}(M)-2 M a_{3}(M)\right)+ \\
& +\lambda^{3} \sigma_{Q}^{2}\left(3 a_{2}^{2}(M)+3 M^{2} a_{1}^{2}(M)-2 a_{1}(M) a_{3}(M)\right)+ \\
& +\lambda^{4} \sigma_{Q}^{3} \gamma_{Q} a_{1}^{2}(M) a_{2}(M)+3 \lambda^{4} \sigma_{Q}^{4} a_{1}^{2}(M) a_{2}(M)+\lambda^{5} \sigma_{Q}^{5} \gamma_{Q} a_{1}^{4}(M)+ \\
& +\left(3 \sigma_{Q}-\gamma_{Q}\right) \sigma_{Q}^{3} \lambda^{4}\left(2 M a_{1}^{3}(M)-2 a_{1}^{2}(M) a_{2}(M)\right) .
\end{aligned}
$$

Then,

$$
U^{\prime}(M)=(1-G(M)) D(M),
$$

with

$$
D(M)=z \sigma_{S}^{-1}(M) H(M)+\frac{1}{6}\left(z^{2}-1\right) \sigma_{S}^{-2}(M) P(M)-\alpha \lambda
$$

Noting that (13) and (14) are of the same sign, then given $\mathrm{H}_{2}$, it can be concluded that (13) takes the value zero, for finite $M$, if and only if (14) is zero.

Hence the result will be proved by showing that if (6) holds then

$$
\lim _{M \rightarrow 0^{+}} D(M)<0
$$

and that (7) implies that

$$
\lim _{M \rightarrow+\infty} D(M)=+\infty
$$

Let

$$
\xi_{k}(M)=\frac{a_{k}(M)}{M^{k}}, \quad k=1,2,3
$$

Considering that

$$
0 \leq \int_{0}^{M} \frac{x^{k}}{M^{k}} d G(x) \leq G(M), \quad \forall M>0
$$

then

$$
\lim _{M \rightarrow 0^{+}} \int_{0}^{M} \frac{x^{k}}{M^{k}} d G(x)=0
$$

which implies that

$$
\lim _{M \rightarrow 0^{+}} \xi_{k}(M)=1
$$


Multiplying and dividing the first term of the right hand side of (14) by $M$ and the second by $M^{4}$ and taking the limit using (19), one obtains

$$
\lim _{M \rightarrow 0^{+}} D(M)=z \sqrt{\lambda+\lambda^{2} \sigma_{Q}^{2}}+\frac{1}{6}\left(z^{2}-1\right) \frac{\lambda+3 \lambda^{2} \sigma_{Q}^{2}+\lambda^{3} \sigma_{Q}^{3} \gamma_{Q}}{\lambda+\lambda^{2} \sigma_{Q}^{2}}-\alpha \lambda
$$

which shows that (6) implies (15).

On the other hand, it is obvious that, if $G(x)$ has a finite second moment, the first term of $D(M)$, with $D(M)$ given by (14), tends to $+\infty$ when $M \rightarrow+\infty$. It will be shown that this still happens when the second moment does not exist.

Indeed, given the assumptions about $G(x)$ and using integration by parts

$$
\xi_{k}(M)=\frac{k}{M^{k}} \int_{0}^{M} x^{k-1}(1-G(x)) d x
$$

so,

$$
0 \leq \xi_{2}(M) \leq \frac{2}{M} \int_{0}^{M}(1-G(x)) d x
$$

As

$$
\lim _{M \rightarrow+\infty} \int_{0}^{M}(1-G(x)) d x=\mathrm{E}[X]
$$

which exists by $H_{3}$, then

$$
\lim _{M \rightarrow+\infty} \xi_{2}(M)=0
$$

and

$$
\lim _{M \rightarrow+\infty} \xi_{1}(M)=0
$$

which proves that

$$
\lim _{M \rightarrow+\infty} \sigma_{S}^{-1}(M) H(M)=+\infty
$$

As the first term on the right hand side of (14) tends to $+\infty$ when $M \rightarrow+\infty$, it is sufficient to show that, if (7) holds, the second term can not tend to $-\infty$, to prove (16). For that purpose, note that

$$
M a_{k}(M)>a_{k+1}(M), \quad \forall M>0,
$$

which proves that, if (7) is fulfilled, $P(M)$ given by (12) is positive for all $M>0$.

To finish the proof it is enough to remind that, by assumption, $z>1$. 
Note that (7) holds when $N$ is Poisson ( $Q$ is degenerate), but also when $N$ is Negative Binomial ( $Q$ is Gamma and $\gamma_{Q} / \sigma_{q}=2$ ) and even when $Q$ is Inverse Gaussian (then $\gamma_{Q} / \sigma_{Q}=3$ ). But condition ( 7 ) is far from being a necessary condition. If, for instance, $G$ has a finite third moment, the limit of (12) when $M \rightarrow+\infty$ is $+\infty$ independently of the sign of $3 \sigma_{Q}-\gamma_{Q}$.

Result 2: Using the Normal approximation to the retained risk, $U(M)$ with $M>0$, has a global minimum if and only if

$$
\alpha>=z \frac{\sigma_{N}}{\lambda}
$$

The minimum is attained at the point $M^{*}$, where $M^{*}$ is the unique solution to the equation

$$
z \sigma_{S}^{-1}(M)\left[M+\lambda a_{1}(M) \sigma_{Q}^{2}\right]=\alpha .
$$

\section{Proof of Result 2:}

If the approximation to the Normal distribution is used (2) simplifies to

$$
U(M)=z \sigma_{S}(M)-(P-\mathrm{E}[S]-C(M))
$$

and (14) to

$$
D(M)=z \sigma_{S}^{-1}(M) H(M)-\alpha \lambda,
$$

with $H(M)$ given by (11).

It will be shown that the second derivative of $U(M)$ is positive whenever the first derivative of $U(M)$ is zero for $0<M<+\infty$. Indeed,

$$
\left.U^{\prime \prime}(M)\right|_{U^{\prime}(M)=0}=\left\{z \sigma_{S}^{-3}(M)\left[\lambda^{2} \int_{0}^{M} x^{2} d G(x)+\lambda^{3} \sigma_{Q}^{2} l(M)\right]\right\}(1-G(M))
$$

where

$$
l(M)=a_{1}^{2}(M)+a_{2}(M)(1-G(M))-2 a_{1}(M) M(1-G(M))
$$

To show that (28) is positive for $0<M<+\infty$, it is enough to show that (29) is not negative.

As $l(0)=0$ then

$$
l(M)=\int_{0}^{M} l^{\prime}(w) d w
$$

which is equivalent to

$$
l(M)=\int_{0}^{M}\left(2 w a_{1}(w)-a_{2}(w)\right) d G(w)
$$


As (31) is non-negative for all $w \geq 0$ the same happens to (30), which shows that

$$
\left.U^{\prime \prime}(M)\right|_{U^{\prime}(M)=0}>0, \quad \forall M>0
$$

which implies that there exists at most one finite root of

$$
U^{\prime}(M)=0,
$$

and that when it exists it is an absolute minimum of $U(M)$.

It is a consequence of the proof of Result 1 that such a root exists if and only if (24) holds (note that (7) was only used to show that the second term on the right hand side of (14) can not be negative).

Note that if $N$ is Poisson distributed, (6) and (24) are equivalent respectively to

$$
\alpha>\frac{z}{\sqrt{\lambda}}+\frac{z^{2}-1}{6 \lambda}
$$

and

$$
\alpha>\frac{z}{\sqrt{\lambda}} .
$$

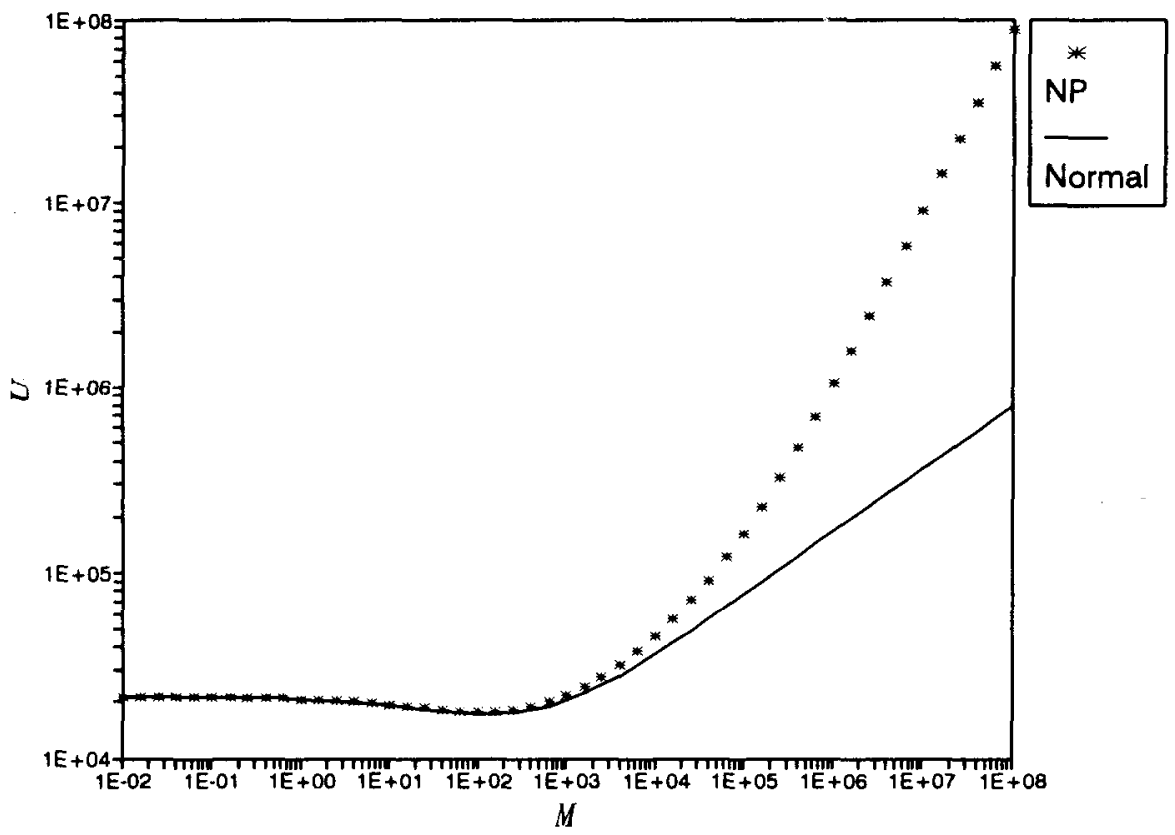

Figure 1. The initial reserve as a function of $M$. Mon. Unit: 1000 Pounds. 


\section{AN EXAMPLE}

Figure 1 shows $U(M)$ when the Normal Power and the Normal approximation are used, for the data used by Beard, Pentikäinen and Pesonen (1984), with the exception of the loading coefficient, which is here taking the value 0.25 , i.e. for

$$
\begin{aligned}
& \varepsilon=0.001 \\
& \lambda=10000 \\
& P=1.04 \lambda \mathrm{E}[X] \\
& \sigma_{Q}=0.038 \\
& \gamma_{Q}=0.25 \\
& \alpha=0.25
\end{aligned}
$$

and $G$ according to Table 3.5.1. of Beard, Pentikäinen and Pesonen (1984), pp. 62-63 (columns 2 and 4). The minimum of $U(M)$ when the NP approximation is used is attained at $M^{*}=100$ thousand pounds, and it is slightly higher when the Normal approximation is used.

Note that one does not expect big changes on the behaviour of the function $U(M)$ when each of the approximations is used, since for reasonable values of $M$, the second term on the right hand side of equation (2) is relatively small, becoming significant only for large values of $M$.

Having in mind the results obtained one can conclude that the formulation of the retention problem in such a way that the initial reserve necessary to assure with probability $1-\varepsilon$ that it is not absorbed during the period under consideration is reduced to a minimum, makes sense.

Conditions (6) and (24) are not fulfilled only when the loading coefficient used by the reinsurer is very small or when the required probability is very high.

As $M^{*}$ is a function of the required probability, it would be advisable, in a practical situation, to study $M^{*}$ as a function of $z$.

\section{REFERENCES}

Beard, R. E., Pentikälnen, T. and Pesonen, E. (1984) Risk Theory. Chapman and Hall, London, third edition.

Daykin, C. D., Pentikäinen, T. and Pesonen, M. (1994) Practical Risk Theory for Actuaries. Chapman and Hall, London.

Maria de Lourdes Centeno

Instituto Superior de Economia e Gestão

Universidade Técnica de Lisboa

Rua Miguel Lupi, 20

1200 Lisboa 